\title{
Non-autonomous Logistic Equations and Optimization of Renewable Resources Management
}

\author{
Mingli Zhong \\ University of Rochester \\ mzhong5@u.rochester.edu \\ Advisor: Nsoki Mavinga \\ Swarthmore College \\ mavinga@swarthmore.edu
}

\begin{abstract}
This paper concerns applications of the non-autonomous logistic equation to bioeconomic fishery models. We develop a generalized model which satisfies both biological and economic principles of optimization without overexploitation. We also provide conditions to optimize the revenue and to maximize the sustainable yield.
\end{abstract}




\section{Introduction}

The autonomous logistic equation was first introduced by Pierre F. Verhulst in 1838 to study the dynamics of human populations with self-limitations [11]. Before Verhulst, researchers simply assumed that the rate of a population growth depends on the size of the population, which is given by the following equation

$$
\frac{d P}{d t}=r P, P(t)=P_{0} e^{r t}
$$

where $P$ represents the population at time $t$ (e.g. [5]). Verhulst noticed that the exponential growth of a population was unrealistic over a long-term period even though it depicted the growth of some populations over a relatively short-term interval. He introduced a new population model given by the logistic equation:

$$
\frac{d P}{d t}=r\left(1-\frac{P}{K}\right) P
$$

where $r$, the intrinsic growth rate, and $K$, the environmental carrying capacity, are constants. A significant improvement from the exponential model to Eq. (1) is the self limitation component, which denies the possibility of unlimited exponential growth. In addition to the population model, the autonomous logistic function is widely used to predict the spread of contagious diseases (see e.g [2] [5]), such as H1N1. It is also used to build bioeconomic models for natural resource management (see e.g [4]).

In this paper, we are interested in the non-autonomous logistic equation, given by the equation

$$
\frac{d u}{d t}=u(a(t)-b(t) u), \quad t \in \mathbb{R}
$$

where the carrying capacity $a: \mathbb{R} \rightarrow \mathbb{R}$ and the self-limitation coefficient $b: \mathbb{R} \rightarrow \mathbb{R}$ are positive and continuous functions satisfying the following constraints:

$$
0<\alpha \leq a(t) \leq A, \quad 0<\beta \leq b(t) \leq B, \quad t \in \mathbb{R} .
$$

While Eq. (1) can be solved explicitly by employing classical techniques, Eq. (2) becomes more complicated to solve. However, it is a more realistic model because self-limitation and carrying capacity fluctuate over time (see e.g [8]).

The purpose of this paper is to study the non-autonomous logistic bioeconomic models, such as fishery models, and to provide conditions to optimize the revenue and to maximize the sustainable 
yield, which is the maximum use that an ecological resource can sustain without impairing its renewability through natural growth [1]. This paper is organized as follows: In Section 2, we give an explicit proof of the existence and uniqueness of the solution to Eq. (2), which was first proved by Nkashama in [7], as a basis of our bioeconomic models. The proof shows that the solution is bounded, positive, and does not tend to zero in the past or in the future. In Section 3, we develop the generalized non-autonomous logistic bioeconomic model and the maximum sustainable yield in terms of the relationship between natural growth and harvesting by valuing natural resource as a stock. We also present the non-autonomous optimal fishery models by combining the biological and economic models. This is the first time that the non-autonomous logistic equation has been considered in the case of optimal fishery models. In addition, we interpret the disadvantage of overexploitation in three ways: first, when the sustainable yield is below a maximum value, it causes the population to approach zero; second, when the total cost is higher than the total revenue, economic overfishing implies economic inefficiency; third, overexploitation consumes the future value of biological resource.

\section{Theoretical background of non-autonomous logistic equation}

In this section, we prove the existence and uniqueness of bounded solutions of Eq. (2). In [7], Nkashama proved for the first time that there exists a unique, bounded and positive solution of Eq. (2) that does not tend to zero in the past or in the future. We would like to elaborate and give more details in the proof of Nkashama.

We obtain a quantitative estimate of the blow-up time in terms of the initial condition and bounds in (3). By blow up time we mean the following:

Definition 2.1 [10]. Let $\varphi\left(t, x_{0}\right)$ be the solution to the initial value problem be $\dot{x}=p \circ x, x(0)=$ $x_{0}$ (with $\left.p \in C^{1}\left(\mathbb{R}^{n}, \mathbb{R}^{n}\right), x_{0} \in \mathbb{R}^{n}\right)$ and where $t \in I$ with some interval $I \subset \mathbb{R}$ containing 0 . $\varphi\left(t, x_{0}\right)$ is said to blow up if there exists $t_{*} \in \mathbb{R}^{+}$such that for all $M \in \mathbb{R}$, there exists $\in \in \mathbb{R}^{+}$for which and for all $t<t_{*}$ such that $t_{*}-t<\epsilon$ the inequality $\left\|\varphi\left(t ; x_{0}\right)\right\| \geqslant M$ holds.

Theorem 2.2 [7] Suppose that the ineq. (3) hold. Then Eq. (2) has exactly one bounded solution $u: \mathbb{R} \rightarrow \mathbb{R}$ that is positive, and that does not tend to zero as $t \rightarrow \pm \infty$. Actually, $u(t)$ satisfies the inequalities 


$$
\frac{\alpha}{B} \leq u(t) \leq \frac{A}{\beta}
$$

for all $t \in \mathbb{R}$.

Proof. Observe that $u=0$ satisfies Eq. (2). By the uniqueness of solutions to the initial-value problem, any non-trivial solution to Eq. (2) must be either positive or negative on its interval of definition. We will prove the theorem in three steps.

Step 1. Suppose that $u(t)$ is a non-trivial solution to Eq. (2) such that $u$ is bounded on $\mathbb{R}$. We will show that $u$ must satisfy the inequalities

$$
0<u(t)<\frac{A}{\beta}
$$

for all $t \in \mathbb{R}$.

Indeed, suppose by contradiction that $u\left(t_{0}\right)<0$ for some $t_{0}$, then $u(t)$ is negative and decreasing for all $t \in \mathbb{R}$ for which $u(t)$ is defined. Therefore, from Eq. (2) and conditions (3), we have that

$$
\frac{d u}{d t} \leq \alpha u-\beta u^{2}<0 \Rightarrow\left(\alpha u-\beta u^{2}\right)^{-1} \frac{d u}{d t} \geq 1 .
$$

Using partial fractions, we obtain

$$
\frac{d}{d t} \ln \left(\frac{\alpha u}{\beta u-\alpha}\right) \geq \alpha .
$$

Integrating from $t_{0}$ to $t$, with $t_{0} \leq t$, we get

$$
\ln \left(\frac{\alpha u(t)}{\beta u(t)-\alpha}\right)\left(\frac{\beta u\left(t_{0}\right)-\alpha}{\alpha u\left(t_{0}\right)}\right) \geq \alpha\left(t-t_{0}\right)
$$

Set $c_{0}=\alpha u\left(t_{0}\right) /\left(\beta u\left(t_{0}\right)-\alpha\right)$, then

$$
\begin{gathered}
u(t)\left(\alpha-\beta c_{0} e^{\alpha\left(t-t_{0}\right)}\right) \geq-\alpha c_{0} e^{\alpha\left(t-t_{0}\right)}, \text { and } \\
u(t) \leq \frac{c_{0} \alpha}{c_{0} \beta-\alpha e^{-\alpha\left(t-t_{0}\right)}} .
\end{gathered}
$$


Notice that the vertical asymptote of the solution curve is given by

$$
t_{*}=t_{0}+\alpha^{-1} \ln \left[\alpha\left(\beta c_{0}\right)^{(-1)}\right]
$$

Therefore, $u\left(t_{*}\right)=c_{0} \beta-\alpha e^{-\alpha\left(t-t_{0}\right)}=0$.

Observe that if $t_{0}<t<t_{*}$, we have that

$$
\begin{gathered}
c_{0} \beta-\alpha e^{-\alpha\left(t-t_{0}\right)}<c_{0} \beta-\alpha e^{-\alpha\left(t_{*}-t_{0}\right)}=0 \\
c_{0} \beta-\alpha e^{-\alpha\left(t-t_{0}\right)}<0 .
\end{gathered}
$$

In addition, $c_{0} \alpha>0$, so the right side of Eq. (6) is negative. It follows that $u(t) \rightarrow-\infty$ as $t \rightarrow t_{*}^{-}$. In other words, $u(t)$ blows up in finite time forward. This contradicts the fact that $u(t)$ is bounded. Thus, $u(t)$ must be positive.

Now we would like to prove that $u(t)<A / \beta$ for all $t \in \mathbb{R}$. Suppose by contradiction that $u(t) \geq A / \beta$ for some $t_{0} \in \mathbb{R}$, then $u(t)$ is decreasing for all $t \leq t_{0}$ for which $u(t)$ is defined. Therefore, from Eq. (2) and conditions (3), we have that

$$
d u / d t \leq A u-\beta u^{2}<0 \Rightarrow\left(A u-\beta u^{2}\right)^{-1} d u / d t \geq 1
$$

Using partial fractions technique, we obtain

$$
\frac{d}{d t} \ln \left(\frac{A u}{\beta u-A}\right) \geq A .
$$

Integrating from $t$ to $t_{0}$, with $t \leq t_{0}$, we get that

$$
\ln \left(\frac{A u\left(t_{0}\right)}{\beta u\left(t_{0}\right)-A}\right)\left(\frac{\beta u(t)-A}{A u(t)}\right) \geq A\left(t_{0}-t\right) .
$$

Set $c_{0}=\frac{A u\left(t_{0}\right)}{\beta u\left(t_{0}\right)-A}$, then

$$
\begin{gathered}
u(t)\left(A-\beta c_{0} e^{A\left(t_{0}-t\right)}\right) \geq-A c_{0} e^{A\left(t_{0}-t\right)}, \text { and } \\
u(t) \geq \frac{c_{0} A}{c_{0} \beta-A e^{-A\left(t_{0}-t\right)}} .
\end{gathered}
$$

Observe that the vertical asymptote of the solution curve is

$$
t_{*}=t_{0}+A^{-1} \ln \left[A\left(\beta c_{0}\right)^{(-1)}\right]
$$


Therefore, $u\left(t_{*}\right)=c_{0} \beta-A e^{-A\left(t_{0}-t\right)}=0$.

Since $t_{*}<t<t_{0}$, we have

$$
\begin{gathered}
c_{0} \beta-A e^{-A\left(t_{0}-t\right)}>c_{0} \beta-A e^{-A\left(t_{*}-t_{0}\right)}=0 \\
\Rightarrow c_{0} \beta-A e^{-A\left(t_{0}-t\right)}>0 .
\end{gathered}
$$

In addition, $c_{0} A>0$, so the right side of Eq. (8) is positive. It follows that $u(t) \rightarrow \infty$ as $t \rightarrow t_{*}^{+}$. In other words, $u(t)$ blows up in finite time backward. This contradicts the fact that $u(t)$ is bounded. Thus, the inequality (4) must hold for every bounded solution to Eq. (2). One can see that (7) and (9) give us quantitative estimates of the blow-up time in terms of the initial condition and the bounds.

Step 2. We want to prove that there exists at least one bounded solution for Eq. (2). Assume that $\varepsilon \in \mathbb{R}$ such that $0<\varepsilon<\alpha / B$. Consider the initial-value problem

$$
\left\{\begin{array}{l}
\frac{d w}{d t}=w(a(t)-b(t) w) \quad t \in \mathbb{R} \\
w\left(t_{0}\right)=\varepsilon
\end{array}\right.
$$

Since $w(a(t)-b(t) w)$ is continuous then by the existence results of initial value problem, there exists a solution $w$ of problem (10) defined on $\mathbb{R}$. Now we claim that $w$ also satisfies inequalities in (5). Indeed, $w^{-1} d w / d t>\varepsilon_{2}$ for all $t \leq t_{0}$, where $\varepsilon_{2}=\alpha-B \varepsilon>0$. Integrating from $t$ to $t_{0}$, we obtain that:

$$
\begin{aligned}
& \ln \frac{w\left(t_{0}\right)}{w(t)} \geq \varepsilon_{2}\left(t_{0}-t\right) \\
& w(t) \leq w\left(t_{0}\right) \frac{1}{e^{\varepsilon_{2}\left(t_{0}-t\right)}} .
\end{aligned}
$$

Therefore,

$$
0<w(t) \leq w\left(t_{0}\right) e^{\varepsilon_{2}\left(t-t_{0}\right)} \text { for all } t \leq t_{0}
$$

This implies that $w(t)$ can be continued indefinitely in the past, and $w(t) \rightarrow 0$ as $t \rightarrow-\infty$. Now, let us show that $0<w(t) \leq A / \beta$ for all $t \geq t_{0}$ and $w(t)$ can be continued indefinitely in the future. Suppose by contradiction that $w\left(t_{1}\right)>A / \beta$ for some $t_{1}>t_{0}$. Since $w\left(t_{0}\right)<\alpha / B$, then by the Intermediate Value Theorem and the fact that $w(t)$ is decreasing, hence $w\left(t_{1}\right)<\alpha / B$, which lead to a contradiction since $\alpha / B<A / \beta$. Thus, $w(t)$ is between 0 to $A / \beta$. 
Now, let us show that there exists at least one solution for Eq. (2). Indeed, let $I \subset \mathbb{R}$ be defined by

$$
I=\left\{w_{0} \in \mathbb{R}: \text { Eq. (1), with } u(0)=w_{0} \text {, has a bounded solution }\right\} .
$$

Notice that $I$ is not empty since $u(0)=w_{0}=0$ is in $I$.

Set $u_{0}=\sup I$, consider the following equation

$$
\left\{\begin{array}{l}
\frac{d u}{d t}=u(t)(a(t)-b(t) u(t)) \quad t \in \mathbb{R} \\
u(0)=u_{0}
\end{array}\right.
$$

We claim that $\alpha / B \leq u_{0} \leq A / \beta$. Indeed, if $u_{0}<\alpha / B$ then arguing as above, we see that there exists a solution to problem (12) bigger than $u_{0}$, which contradicts the fact that $u_{0}$ is the supremum. Therefore, $u_{0} \geq \alpha / B$. Now, if $u_{0}>A / \beta$, pick $w_{0} \in \mathbb{R}$ such that $A / \beta<w_{0}<u_{0}$. Using (8), we have that

$$
w(t) \geq \frac{c_{0} A}{c_{0} \beta-A e^{A t}} .
$$

From step 1, $w(t) \rightarrow \infty$ as $t \rightarrow-\infty$. Hence, $w(t)$ blows up in finite time in the past, which violates the fact that $u_{0}$ is the supremum of initial conditions of bounded solutions. So, $u(t) \leq A / \beta$ for all $t \in \mathbb{R}$. Thus there exists a solution $u(t)$ satisfying inequality (5).

Now let us show that $u(t) \geq \alpha / B$ for all $t \leq 0$. Indeed, suppose by contradiction that $u\left(t_{0}\right)<\alpha / B$ for some $t_{0}<0$. Pick $\varepsilon \in \mathbb{R}$ such that $u\left(t_{0}\right)<\varepsilon<\alpha / B$. Then the solution to Eq. (10) is bounded on $\mathbb{R}$, with $w(0)>u_{0}$ by uniqueness of solution to initial-value problems. This contradicts the fact that $u_{0}$ is the supremum. So, $u(t) \leq \alpha / B$ for all $t \in \mathbb{R}$. Thus Eq. (1) has the maximal solution $u(t)$ satisfying the inequality (4).

Step 3. We need to show that there exists a unique and bounded solution to Eq. (2) that satisfies Eq. (5). Let $J \subset I$ be defined by

$$
J=\left\{w_{0} \in \mathbb{R}: w_{0} \in I \text { and (5) holds. }\right\}
$$

Observe that $J$ is not empty, since $\alpha / B \leq u_{0} \leq A / \beta, u_{0} \in I$.

Set $v_{0}=\inf J$. We have that $v(0)=v_{0}$. Since $u_{0} \in J$ and $v_{0}$ is the infimum, $v_{0}<u_{0}$. Hence $\alpha / B \leq v_{0} \leq u_{0} \leq A / \beta$. Using similar argument as in step 2, the minimal solution $v(t)$ also satisfies inequality (5). Hence

$$
0<\frac{\alpha}{B} \leq v(t) \leq u(t) \leq \frac{A}{\beta} \text { for all } t \in \mathbb{R}
$$


Now, we proceed by showing that $v(t)=u(t)$ for all $t \in \mathbb{R}$. Suppose by contradiction that $v(t)<u(t)$ for all $t \in \mathbb{R}$, then

$$
\begin{gathered}
\left\{\begin{array}{l}
\frac{d v}{d t}=v(a(t)-b(t) v) \\
\frac{d u}{d t}=u(a(t)-b(t) u)
\end{array}\right. \\
\frac{1}{v} \frac{d v}{d u}-\frac{1}{u} \frac{d v}{d t} \geq b(t)(u(t)-v(t)) \\
\frac{d}{d t}\left(\ln \left(\frac{v}{u}\right)\right) \geq b(t)(u(t)-v(t))>0 .
\end{gathered}
$$

Hence $\ln (v / u)$ is increasing.

$$
\begin{aligned}
& \frac{v(t)}{u(t)}\left(\frac{v(t)}{u(t)}\right)^{\prime} \geq b(t)(u(t)-v(t))>0 \\
& \left(\frac{v(t)}{u(t)}\right)^{\prime} \geq \frac{u(t)}{v(t)} b(t)(u(t)-v(t))>0 .
\end{aligned}
$$

Hence $v / u$ is increasing. Then

$$
\frac{v(t)}{u(t)} \leq \frac{v(0)}{u(0)} \leq c<1 \text { for all } t \leq 0
$$

Since $u(t) \leq \alpha / B$

$$
u(t)-v(t) \geq(1-c) u(t) \geq(1-c) \frac{\alpha}{B}=\delta>0 \text { for all } t \leq 0
$$

Integrating Eq. (14) from $t$ to 0 , with $t \leq 0$, we obtain

$$
\begin{gathered}
\ln \frac{v_{0}}{u_{0}}-\ln \frac{v(t)}{u(t)} \geq-\beta \delta t \\
0<\frac{v(t)}{u(t)} \leq e^{\beta \delta t} \frac{v_{0}}{u_{0}} \text { for all } t \leq 0 .
\end{gathered}
$$

Hence, when $t \rightarrow-\infty, v(t)-u(t) \rightarrow 0$. This contradicts the fact that $v(t) / u(t) \geq \alpha \beta / A B>0$ for all $t \in \mathbb{R}$. Therefore, $v(t)=u(t)$.

In conclusion, we prove that the logistic equation with positive non-autonomous bounded coefficients has exactly one bounded solution that is positive and that does not approach the zerosolution in the past and in the future. In a quantitative perspective, we obtain an estimate of the blow-up time in terms of the initial condition and the bounds in (3). 


\section{Empirical bioeconomic models}

In this section, we develop a non-autonomous model which formulates a dynamic theory of renewableresource management. First we give some preliminary concepts that will be used later. Bioeconomics is the study of the dynamics of biological resources. Resource management has increasingly become a necessity for sustainable development and the long-term benefit of humanity. Bioeconomists attempt to use mathematical and economic models to optimally manage renewable resources [4].

The biological resources cannot be exploited too heavily without an ultimate loss of productivity. Balance between the surplus production and sustainable yield is the perfect condition.

Now we consider the following dynamic model for the exploitation of biological resources (see.g.[4])

$$
\frac{d x}{d t}=F(x)-h(t)
$$

where $F(x)$ represents the natural growth rate of the population and $h(t)$ represents the rate of harvesting. When $h(t)=F(x)$, the population reaches the equilibrium point. At that level, the natural growth rate $F(x)$ also equals the sustainable yield that can be harvested while maintaining a fixed population level $x$. When $h(t)$ is not equal to $F(x)$, the system is not in equilibrium. In many cases, equilibrium solutions emerge from the dynamic theory.

\subsection{Generalized non-autonomous logistic bioeconomic model}

Suppose that there is no human intervention, such as harvesting. The population $x$ does not grow exponentially due to the limitations of space, water, and other natural resources. Then $F(x)$ can be simplified as an autonomous logistic model. Suppose that in a certain population, the birth rate is $b$ and the mortality rate is $m$. Let us denote $r=b-m$. Since $h(t)=0$, we have the following logistic equation

$$
\frac{d x}{d t}=F(x)=r x\left(1-\frac{x}{K}\right), \quad x(0)=x_{0},
$$

where the constant $r$ is the intrinsic growth rate, and the positive constant $K$ is the environmental saturation level. Observe that there are two equilibrium solutions to the Eq. $(16): x(t) \equiv 0$ 
and $x(t) \equiv K$. A non-trivial solution to Eq. (16) is given by

$$
x(t)=\frac{K}{1+c e^{-r t}},
$$

where $c=\frac{K-x_{0}}{x_{0}}$.

If $x(0)>0$, and $x(t)<K$, then $d x / d t>0$, therefore $\lim _{t \rightarrow \infty} x(t)=K$; If $x(t)>K$, then $d x / d t<0$. Therefore, $\lim _{t \rightarrow \infty} x(t)=K$. Thus, $K$ is a stable equilibrium.

Now assume that $h(t) \neq 0$, and it is considered as the rate of removal. Suppose that $h(t)$ is constant. Consider

$$
\frac{d x}{d t}=F(x)-h=r x\left(1-\frac{x}{K}\right)-h .
$$

Observe that $\frac{1}{4} r K$ is the maximum value of $F(x)$. The relationships between $\max F(x)$ and $h$ are as follows:

When $h<\frac{1}{4} r K$, we have that the generalized model possesses two equilibria, $x_{1}$ and $x_{2}$. At these two points, $h$ reaches its sustainable yield, so the solution $x(t)$ is not decreasing or increasing at both points. However, neither $x_{1}$ nor $x_{2}$ is the maximum level of sustainable yield.

When $h=\frac{1}{4} r K$, there is single equilibrium at $x=K / 2$. At that time, $h$ reaches its maximum sustainable yield (MSY). $h_{M S Y}=\max F(x)$ with $x_{M S Y}=K / 2$.

When $h>\frac{1}{4} r K, x(t)$ is decreasing at all times and approaches 0 for any initial level $x(0)$. This is the mathematical interpretation of overexploitation. When $x(t)$ is below the maximum sustainable yield due to some degree of overexploitation, the only way to recover the population is to reduce the harvest rate $h$ below MSY to reach the corresponding value of $d x / d t$ in terms of existing $x(t)$.

We are interested in the case when $r$ is no longer a constant. We consider the following equation:

$$
\frac{d x}{d t}=r(t) x\left(1-\frac{x}{K(t)}\right)-h,
$$

where $r(t)$ represents the carrying capacity and $r(t) / K(t)$ represents the self-limitations. Suppose $0<\alpha \leq r(t) \leq A$, and $0<\omega \leq K(t) \leq W$, then

$$
\beta=\frac{\alpha}{W} \leq \frac{r(t)}{K(t)} \leq \frac{A}{\omega}=B
$$

From section 2, there exists a bounded solution that does not tend to zero in the past or in the future. Figure 1 shows a particular case when $K$ is assumed to be constant. 
In summary, the relationships between the natural growth rate and the rate of removal indicate two facts: first, the maximum sustainable yield, the ideal condition that provides human beings the highest productions without exploiting the environment, can only be reached when the maximum growth rate equals the rate of removal; second, in terms of its mathematical interpretation, overexploitation implies a resource reduced to a level below the maximum sustainable yield that $x$ is below $x_{M S Y}$. (The definition of maximum sustainable yield can be found in [4].)

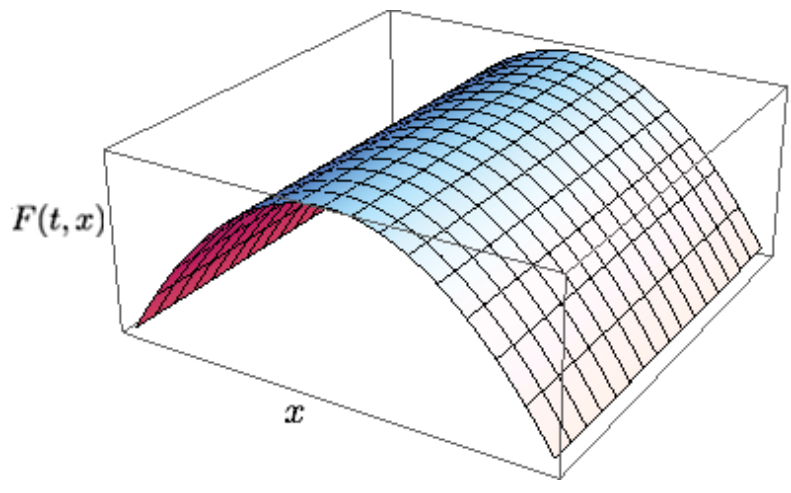

Figure 1: $F(t, x)=r(t) x\left(1-\frac{x}{K}\right)-h$, assuming that $\mathrm{K}$ is constant

\subsection{Optimal fishery model based on economic theories}

In this section, we consider a fishing model in bioeconomics. The unit of measurement is the total number of vessel-days per unit time. Formally, it is measured by catch-per-unit-effort (CPUE) (see [9]). Catch-per-unit effort (CPUE) is an indirect measure of the abundance of a target species, such as in commercial fisheries. Changes in the catch-per-unit effort are inferred to signify changes in the target species' true abundance. A decreasing CPUE indicates overexploitation, while an unchanging CPUE indicates sustainable harvesting.

Since CPUE is proportional to the current stock level of the fish population (more effort, more fish), the catch rate $h$ can be expressed as follows:

$$
h(t)=q E x(t)
$$

where the constant $E$ is the effort and the constant $q$ represents the catchability coefficient, which is measured by per vessel day. $x(t)$ is the fish population at time $t$. The logistic growth model for fisheries is given by: 


$$
\frac{d x}{d t}=r x\left(1-\frac{x}{K}\right)-q E x
$$

Figure 2 shows when $E$ and $r$ are both constant. Note that the equilibrium value is $x_{1}=$ $K(1-q E / r)$.

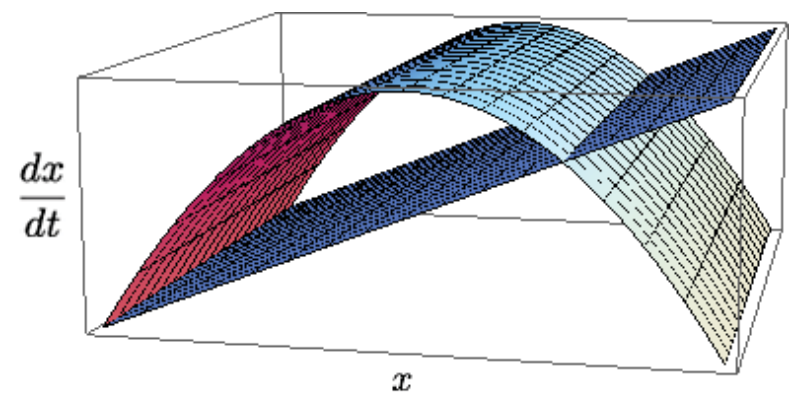

Figure 2: $\frac{d x}{d t}=(r-q E) x-\frac{r}{K} x^{2} . r$ and $E$ are constant.

The sustainable yield at that time is $Y=h=q E x_{1}=q E K(1-q E / r)$. In practice, sustainable yield is positive, so $q E / r$ is less than 1 , which means $E<r / q$. In terms of the relationship between $E$ and sustainable yield, to reach the maximum sustainable yield, $E$ and other variables satisfy with the following equations:

$$
\begin{gathered}
q E x_{1}=q E K\left(1-\frac{q E}{r}\right) . \\
h_{M S Y}=\frac{r K}{4}, \text { when } E=\frac{r}{2 q}, x_{1}=\frac{K}{2} .
\end{gathered}
$$

This is compatible to our result in 3.1. Therefore, maximum sustainable yield is the same no matter what interpretation $h(t)$ has as long as the logistic growth model is applied. In the shortterm, the catch rate $h(t)$ is constantly increasing since $h(t)=q E x$ even though $E>r(t) / 2 q$. However, in the long run, $Y$ might decrease in terms of different levels of $E$. Specifically, if $E>r(t) / 2 q$, overexploitation happens, so in the long run, fish population decreases.

When $E$ and $r$ are not constant, a new non-autonomous logistic model is generated:

$$
\frac{d x}{d t}=F(x)-q E(t) x=(r(t)-q E(t)) x-\frac{r(t)}{K} x^{2} .
$$

Observe that the carrying capacity is $r(t)-q E(t)$. It is positive and bounded whenever $r(t)$ is bounded, unless the sustainable yield is zero. Moreover, the fish population approaches zero. 
Before modeling the optimal fishery management, we need to switch from the biological view to an economic one. Capital-theoretic aspects of optimal renewable-resource management aim to concentrate on the model of capital growth and consumption [3]. Based on the satisfaction of human needs, a resource stock is simply a particular form of capital that can either be consumed or conserved. The distinction between a biological resource and a stock of traditional capital, such as machines, is the mechanism of growth. The biological resources grow naturally but traditional capital can only increase through human effort. One fundamental economic theory is that the maximum profit is obtained under the condition of the marginal revenue is equal to the marginal cost. This profit maximization theory still works for common-property resources such as fishery.

Sometimes the exploitation of common-property resources is uncontrolled. However, the fact is that most resources are controlled by authorities partially because of some economic reasons. The Gorden-Schaefer model (Figure 3) explicitly explains these economic reasons:

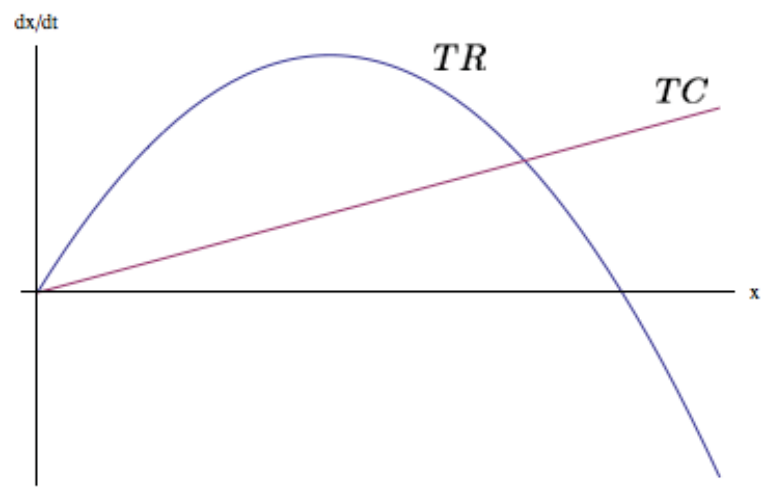

Figure 3: Gorden-Schaefer model. The intersection is the equilibrium $E_{\infty}$.

$T R=p Y(E)$, where $p$ is the price of fish. $T R$ is usually called total sustainable revenue, proportional to $E$. $T C=c E$ is total sustainable cost, where $c$ is constant. Equilibrium value $E_{\infty}$ exists when $T R=T C$, then in terms of Eq. (16) and $T R-T C=p q E x-c E=0$, we get

$$
E_{\infty}=\frac{r}{q}\left(1-\frac{c}{p q K}\right)
$$

$E_{\infty}$ is called bionomic equilibrium since it combines both biological and economic theories. The corresponding stock level $x_{\infty}=c / p q$. An economic interpretation of overexploitation is when $T R<T C$. This is why common-property resources are controlled to reach the maximum profit. 
However, purely focusing on the TR-TC relationship still causes economic inefficiency. Because of the advanced technology, the cost of fishing reduces greatly so that an increasing number of people go to the fishery industry and an excessive level of effort is being utilized. Cheap cost causes economic overfishing. Recall Eq. (22). When $c>p q K$, fishing effort approaches zero, which means that fishing cost is high. It is realistic for some fish species that there are no commercial fishery for them because their market value is far lower than the expense of catching them. When $p q K / 2<c<p q K$, economic overfishing does not occur, and $E_{\infty}$ is lower than $E_{M S Y}$ under this condition. When $0<c<p q K / 2$, there exists the economic overfishing. At this time, $E_{\infty}>E_{M S Y}$. This is what usually happens in reality.

Since the bionomic equilibrium level of $E_{\infty}$ is inefficient, how can the optimum effort level and the optimum sustainable yield be achieved? One method to achieve the optimum level is to maximize $T R-T C$. However, both the economic and biological processes are dynamic. One static situation cannot be kept in one level. An alternative way is to reduce the level of fishing effort. In terms of $Y=q E x$, the immediate impact on $Y$ is to decrease, then ultimately, sustainable yield increases to achieve the maximum $Y$. Fishing effort is smoothly decreasing before reaching the maximum value. Stock level $x=c / p q$, so before achieving maximum sustainable yield, the stock level keeps constant. After the short-term, since $Y$ increases and $E$ keeps constant, $x$ smoothly increases in terms of the relationship between $Y$ and $q E x$.

Rather than maximize $T R-T C$, reducing the fishing effort to achieve maximum sustainable effort is more practical and reasonable. The effective way to reduce the fishing effort is to increase the cost of fishing, such as imposing a fishing tax. Then fewer people participate, total fishing effort decreases, and the sustainable yield increases to its maximum.

\subsection{Non-autonomous optimal fishery model}

The value of a common-property resource is the maximum present value under the consideration of expected future revenue. Natural resource stocks have productive potential in the future and unregulated exploitation disregards future productivity. That is the economic aspect of preventing overexploitation [6].

Given a $P$ value of the reserved fishing resource, its value increases exponentially according to the economic theorem. Assume $\delta=\ln (1+i)$ and $i$ represents the interest rate of the value of 


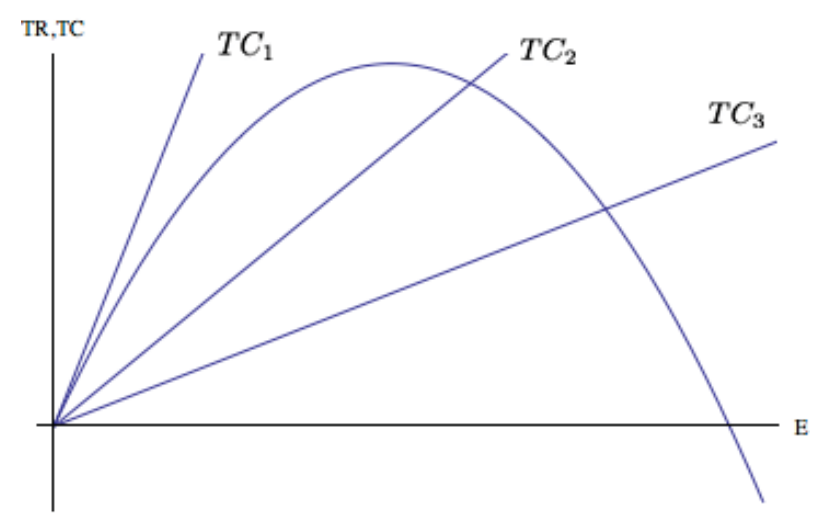

Figure 4: Bionomic equilibrium level $E_{\infty}$ corresponding to different levels of TC.The intersections of $T C_{2}$ and $T R$, and $T C_{3}$ and $T R$ indicate the situation of overexploitation since they are at the right side of the equilibrium point.

fishing resource, then

$$
\begin{aligned}
\text { Future value } & =P e^{(\delta t)} \\
\text { Present value } & =P e^{(-\delta t)},
\end{aligned}
$$

where $\delta$ is the instantaneous annual rate of discount. Discount value and interest value is synonymous when future payments are discounted or when present payments are compounded. Then the total present value (PV) of a sequence of payments $P_{0}, P_{1}, \ldots, P_{N}$,due in years $0,1, \ldots$, $N$, is

$$
\mathrm{PV}=\sum_{K=0}^{N} \frac{P_{K}}{(1+i)^{K}}
$$

In terms of an econometric concept, if the present value is a continuous time-stream of revenues $P(t)$ from zero to $T$, then

$$
\mathbf{P V}=\int_{0}^{T} P(t) e^{-\delta t} d t
$$

This present value interpretation will be applied later in the non-autonomous optimal fishery model. Recall that the natural growth model in the previous section was given by:

$$
\frac{d x}{d t}=F(x)-h(t), t \geqslant 0,
$$


where $h$ is associated with two variables, $x=x(t)$ and $E=E(t)$. Denote $h=a E^{\alpha} x^{\beta}$ and $a, \alpha$, and $\beta$ are all positive constants [6]. For mathematical reasons, set $\alpha=1$ so that $\mathrm{E}$ is linear in the optimization model. Replace $a x^{\beta}$ with $G(x)$ and $h=G(x) E$. Since $x$ is positive, $G(x)$ is non-zero. Suppose the price $P$ of fish is constant, and the cost of a unit of effort is also constant. Then the revenue of an input of effort $E \triangle t$ is

$$
\begin{aligned}
R & \triangle t=R(x, E) \triangle t \\
& =[p-c(x)] h \triangle t
\end{aligned}
$$

where $c(x)=c / G(x)$.

A unit harvest $(h \triangle t=1)$ causes a cost

$$
c E \triangle t=\frac{c}{G(x)} h \triangle t=c(x) h \triangle t=c(x)
$$

Therefore, $c(x)$ is defined as the unit harvesting cost when the population level is $x$. As mentioned before, the optimal model is to maximize the present value under consideration the future value. The objective is transformed mathematically by finding the maximum of

$$
\begin{gathered}
\mathrm{PV}=\int_{0}^{\infty} e^{-\delta t} R(x, E) d t \\
=\int_{0}^{\infty} e^{-\delta t}\{p-c[x(t)]\} h(t) d t,
\end{gathered}
$$

with the constraint that $x(t) \geqslant 0$ and $h(t) \geqslant 0$.

Substitute $h(t)=F(x)-d x / d t$ into Eq. (24), we get

$$
\mathrm{PV}=\int_{0}^{\infty} e^{-\delta t}\{p-c[x(t)]\}\left[F(x)-\frac{d x}{d t}\right] d t
$$

Assume $x=x^{*}$, which is defined as the optimal equilibrium population level:

$$
F^{\prime}\left(x^{*}\right)-\frac{c^{\prime}\left(x^{*}\right) F\left(x^{*}\right)}{p-c\left(x^{*}\right)}=\delta
$$


where $F\left(x^{*}\right)$ is logistic growth model, $c\left(x^{*}\right)$ is the unit harvesting cost when the population level is $x^{*}$, and $P$ is the price of fish. Most-rapid approach aims to achieve $x^{*}$ from any level. Use $h^{*}(t)$ that drives the population $x=x(t)$ toward $x^{*}$ as rapidly as possible:

$$
h^{*}(t)= \begin{cases}h_{\max } & \text { if } x>x^{*} \\ F\left(x^{*}\right) & \text { if } x=x^{*} \\ 0 & \text { if } x<x^{*}\end{cases}
$$

One assumption is that economic parameters remain constant, such as price. The non-autonomous model for $x^{*}=x^{*}(t)$ is:

$$
F^{\prime}\left(x^{*}\right)-\frac{c^{\prime}\left(x^{*}\right) F\left(x^{*}\right)}{p-c\left(x^{*}\right)}=\delta \frac{\frac{d p}{d t}}{p(t)-c\left(x^{*}\right)}
$$

The interpretation of the non-autonomous optimal fishery model is related to marginal productivity of capital. $F(x)$ represents the marginal productivity of the fish population and the second term is a modification of the standard marginal-productivity rule $F\left(x^{*}\right)=\delta$, which $\delta$ is given discount rate. The fact that when the fish population is reduced, the costs of fishing increases. It is reasonable to expect a modification for increased population levels.

Compared to the autonomous optimal model, the additional term at the right side of Eq. (27) is the relative rate of growth of the marginal value of the asset. When $P$ changes, $x$ changes independently from logistic growth. The last concern raised from variable $P$ is about the price effect on the non-autonomous optimal model. When price increases, the effectiveness of value of $\delta$ decreases since $\delta$ is discount rate, so stock level $x^{*}$ increases, which might affect the net future revenue.

\section{Conclusion}

The non-autonomous logistic equation is more realistic to model resource management than the autonomous one. However, the pure logistic equation, either non-autonomous or autonomous, cannot depict bioeconomics comprehensively because of human intervention. Based on the logistic growth equation, the generalized non-autonomous logistic bioeconomic model presents a simplified relationship between natural growth and rate of removal, $d x / d t=F(x)-h(t)$. From the fishery model, we develop the equation to optimize fishing resource management, $d x / d t=$ 
$r x(1-x / K)-q E x$. From this fishery model, a new version of the logistic equation is introduced: $d x / d t=(r-q E(t)) x-r x^{2} / K$, which $E(t)$, varying from time, determines the maximum sustainable yield. Besides the biological view of optimal fishery management, which is to obtain the maximum sustainable yield, the economic aspect is to consider any biological resource as a stock, comparable to buildings and machines. One assumption to optimize profit is that the total revenue equals the total cost. Therefore, the revenue from fishery should be equal to the fishing effort. The fundamental economic idea of the optimal fishing model is to maximize the present value under the consideration of the future revenue. Combining biological and economic theories, the present value model is revised to apply to the optimal fishery model. Since prices of fish vary from time to time, the non-autonomous optimal fishery model considers the relationship between variable $P$ and stock level $x^{*}$.

In addition, there are three ways to interpret overexploitation. When exploitation is uncontrolled, the sustainable yield goes below the maximum value so that the fish population will approach zero. The second way is based on the revenue-cost theory. Overexploitation means more cost than revenue since an overwhelming number of people participate in fishing and the revenue goes lower than what it should be. The third consideration is about the present value and the fu-

ture value. If the fish resource is overexploited today, it implies the loss of future revenue. The maximum present value is based on the protection of the future value.

\section{References}

[1] Glossary of environment statistics. Studies in Methods, F(67), 1997.

[2] H. AMANN. Ordinary Differential Equations: An Introduction to Nonlinear Analysis. Walter de gruyter, 1990.

[3] K. J. ARROW. Optimal capital policy, the cost of capital, and myopic decision rules. Ann. Inst. Statist. Math., (16), 1964.

[4] C. W. CLARK. Mathematical Bioeconomics: The Optimal Management of Renewable Resources. John Wiley \& Sons, 1976. 
[5] W. E. BOYCE \& R. C. DIPRIMA. Elementary Differential Equations and Boundary Value Problems. John Wiley \& Sons, 1997.

[6] C. W. CLARK \& GORDON R. MUNRO. The economics of fishing and modern capital theory: a simplified approach. Journal Of Environmental Economics and Management, (2), 1975.

[7] M. N. NKASHAMA. Dynamics of logistic equations with non-autonomous coefficient. Electron. J. Differential Equations, (02):1-8, 2000.

[8] G. N. GALANIS \& P. K. PALAMIDES. Global positive solutions of a generalized logistic equation with bounded and unbounded coefficients. Electron. J. Differential Equations, (119):1-13, 2003.

[9] W. J. SUTHERLAND. The Conservation Handbook: Research, Management and Policy, chapter Monitoring. Wiley-Blackwell, 2000.

[10] R. CSIJIA \& J. TÓTH. Blow up in polynomial differential equations. World Academy of Science, Enginerring and Techonology, (26), 2007.

[11] P. F. VERHULST. Notice sur la loi que la population poursuit dans son accroissement. Correspondance Mathématique et Physique, 10:113-121, 1838. 